\title{
"Onto-Computer-Project", a Computer Project Domain Ontology : Construction and Validation
}

\author{
Mejri Lassaad ${ }^{1}$ \\ College of Computer and Information Science \\ Jouf University, Saudi Arabia
}

\author{
Hanafi Raja², Henda Hajjami Ben Ghezala ${ }^{3}$ \\ National School of Computer \\ University of Manouba Manouba, Tunisia
}

\begin{abstract}
Ontologies, nowadays, play a primordial role in the representation, the re-use and the sharing of knowledge of a well given domain in a consensual and explicit way more precisely in the computing field. It is in this context that we have proposed a domain ontology baptised onto-computer-project which presents the key to our research goal. This essential goal is to arrive at a final step to elaborate a knowledge based system for computer projects reusing. The aimed system is essentially based on the construction of a memory projects. This memory projects could be defined as a collection of historical and achieved projects around the sphere of computer. This sphere is so wide including many subfields beginning at the database, software engineering fields and arriving at the fields of artificial intelligence, computer vision and so on. This research work requires at first to construct a well-defined ontology in the way to structure and to unify vocabulary often shared by multi actors in the domain of computer projects. To concretize this goal, our paper will describe a construction approach for the proposed domain ontology which is mainly based on an existing methodology named "methontology". The proposed ontology construction approach, which is composed of seven steps, is the result of a comparative study between some ontology construction approaches belonging to different categories of methodology. In fact, we can distinguish four main categories of ontology development approaches: ontology construction approaches from zero, text-based construction approaches, building approaches based on the reuse of already existing ontologies, and crowd souring-based approaches. In our research work, we are interested by the approach of building ontology from zero. Indeed, the construction of the proposed ontology follows an autonomous approach which is not based on any other existing ontology or the updating of an already constructed ontology. In addition, in this paper we are interested by the problem of validating the content of domain ontology and in this context we have proposed an incremental approach for validating the proposed ontology which is composed of six steps. In this context we have studied some ontology validation approaches: those which are questionnaire based, others based on question answering. The problem here that all approaches studied are single actor approaches where a single validation actor can validate the entire ontology and this by applying the semantic and the structural validation definitively with no return. The main originality of our validation approach consists essentially of three criteria: the incremental validation, the multi-intervention, and the respecting of the " $V$ " cycle. In fact, the passage from one validation step to another results in an update of the initial ontology and this by the intervention of three experts (project management expert, a project computer expert and a specialist in ontology engineering). Our proposal approach requires a feedback between all the validation phases and can return to any
\end{abstract}

expert for revalidation if needed. The result of this research is improved a validated ontology which is allowed us to build our project memory and to feed our knowledge base which will serve us to develop our knowledge-based system.

Keywords-Domain ontology; ontology construction; ontology validation; computer project; project memory; knowledge representation

\section{INTRODUCTION}

Ontologies now play a major role in the representation and modeling of knowledge. Their main objective is to formalize the knowledge of a domain and thus add a semantic layer to computer systems and applications. In addition, the development of a new ontology makes it possible to explicitly represent the knowledge of a domain by means of a formal language, in order to be able to be manipulated automatically and shared easily [1].

Indeed, ontology consists of a set of concepts organized using hierarchical and specialized relationships representing a means of expression, sharing and reuse of knowledge, usable by all actors. In addition, an ontology is a computer artifact conceptually modeling knowledge, an indexing system for a specialty area, a theory of scientific content, a representation of shared knowledge or a modeling of reality [2]. It is in this context that we have exploited the proposal of a computer domain ontology to achieve our main research goal which is the capitalization of knowledge from computer project memory. A project memory can be defined as an explicit and continuous representation of knowledge, data or data source within an organization which contains the context in which the knowledge has been created [3]. Therefore, project memory allow professional actors to reuse and share knowledge, which has been capitalized from previous projects in order to carry out a new one [4].

In other hand, the newly created ontology must be validated and evaluated thanks to either experts or standard validation tools. So, we can identify two scenarios [13] which justify the validation of the ontology: an adequate ontology will allow better reuse of the data and ontologists need methods to evaluate and validate their models in order to encourage them to share with confidence their results with the community. It is in this context that this paper will focus primarily on these two aspects: the choice of the methodology of construction of our ontology and the proposition of a validation approach of the proposed ontology. 
The paper is organized as follows. After the introduction, Section 2 consists of the state of the art which is composed of two sub-sections: In the first Sub-section the ontology construction methodologies are reviewed then a comparative study between these methodologies is introduced. In Section 2, we will describe both the main related works of ontology validation approaches and a discussion study. Section 3 presents an ontological construction approach and Section 4 describe the incremental validation approach for our proposed ontology. Finally Section 5 reveals the main conclusion and future works.

\section{RELATED WORKS}

This section consists essentially of two parts: The first one reveals a state of art on the methods of ontological construction and a comparative study of the invoked methodologies. The second one describes the main existing works in the literature and a comparative study of ontology validation approaches. In the following, we introduce the major works in the literature associated with the ontology construction methodologies.

\section{A. Ontology Construction Methodology}

In ontology engineering, the choice of methods, techniques and tools for the ontology construction process is a key step. Indeed, several methodological approaches have been proposed [6][7] to guide this process. We can distinguish four main categories of ontology development approaches: ontology construction approaches from zero [6], text-based construction approaches [8], building approaches based on the reuse of already existing ontologies [10] and crowd souringbased approaches [9].

In our research work, we will be interested in the approach of building ontology from zero. Indeed, the construction of the proposed ontology follows an autonomous approach which is not based on any other existing ontology or the updating of an already constructed ontology. Moreover, the knowledge and skills defining the essential components of the proposed ontology did not come from textual resources.

For all the reasons mentioned above, we found ourselves obliged to adapt the construction approaches from zero for the development of our domain ontology. In the following, we introduce the major works in the literature associated with this kind of approach.

\section{B. Main Approaches from Zero Description}

Several works in the literature are oriented towards this type of approach in what follows we have discussed some proposals.

1) Two-step methodology [11]: As its name indicates, this methodology is composed of two stages: The knowledge organization and the knowledge acquisition and reuse that allow the users collaboratively producing and consuming the knowledge. In the beginning, a Core Reference Ontology [CRO] describing the generic concepts and relations according to the formalized requirements is identified. Then, a Domain Specific Ontology [DSO] is specialized. Only two steps are not enough to describe a complete construction processes. In fact, this methodology is neither documented nor evaluated.

2) On-to-knowledge methodology [OTKM][12]: It is a methodology based on acquired experiences of business activities. It is composed of four stages from identification, to documentation [11]. The stages are given implicitly and the activities are few detailed (general description of the steps and no precision in the choice of components)[3].

3) The methodology proposed by fox \& al [13]: This methodology is used in the context of the TOVE project (Toronto Virtual Enterprise). The application of this methodology is motivated by problems which are formulated under form of informal questions that ontology should answer. This methodology has made it possible to develop complex projects in the field of business but remains limited because neither the different stages nor the techniques used are precisely described.

4) OntoDI methodology [14]: This methodology is an ontology development method which has been developed for the implementation of data integration called ontology development on the data integration domain (OntoDI). The main objective of this ontology construction method is the development of knowledge in ontology to manage the problems of semantic aspects in order to support the implementation of data integration. OntoDI has three main phases: pre-development, main development and postdevelopment. And in each part contains several phases.

5) The methontology [15][16]: It is the most widely used methodology in literature. It is the most adopted construction approach for much ontology in different fields. In fact, this method is highly-precised. "Methontolgy" can be applied in all areas, and it can be applied in scratch or text approach.

In order to adopt an approach to construct our ontology, we will propose a comparative study between the methods already mentioned in the previous sub-section.

\section{Comparative Study}

The comparative study [Table I] is based on four criteria: these criteria are selected in accordance with domain experts.

- Process step: this criterion describes the form or the way in which the construction process steps is defined: detailed, little detailed, very detailed.

- Level of precision: the precision in the choice of the terms, relations and classes during the construction stages. This criterion differs from one method to another.

- Application domain: It serves to know in which domain this method has been applied.

- Type of activity: each process of construction is composed of a set of tasks or activities. Here, we have tried to determine the type of activity. 
TABLE. I. COMPARATIVE STUDY BETWEEN CONSTRUCTION METHODS

\begin{tabular}{|l|l|l|l|l|}
\hline & Process step & $\begin{array}{l}\text { Level of } \\
\text { precision }\end{array}$ & $\begin{array}{l}\text { Applicati } \\
\text { on } \\
\text { domain }\end{array}$ & $\begin{array}{l}\text { Types of } \\
\text { activity }\end{array}$ \\
\hline $\begin{array}{l}\text { Two-step } \\
\text { Methodology } \\
{[11]}\end{array}$ & $\begin{array}{l}\text { Only 2 steps } \\
+ \text { general } \\
\text { description }\end{array}$ & $\begin{array}{l}\text { Generic } \\
\text { concept } \\
+ \text { core } \\
\text { ontology }\end{array}$ & $\begin{array}{l}\text { Knowled } \\
\text { ge } \\
\text { manage } \\
\text { ment }\end{array}$ & Formalisation \\
\hline $\begin{array}{l}\text { OTKM } \\
\text { methodology } \\
{[12]}\end{array}$ & $\begin{array}{l}4 \text { steps+ } \\
\text { detailed } \\
\text { description }\end{array}$ & $\begin{array}{l}\text { Absence of } \\
\text { precision }\end{array}$ & $\begin{array}{l}\text { Business } \\
\text { Domain }\end{array}$ & $\begin{array}{l}\text { Documentatio } \\
\text { n } \\
\text { evaluation }\end{array}$ \\
\hline $\begin{array}{l}\text { Fox and al 's } \\
\text { Methodology } \\
{[13]}\end{array}$ & $\begin{array}{l}4 \text { steps } \\
\text { Not detailed }\end{array}$ & $\begin{array}{l}\text { Lack of } \\
\text { precision }\end{array}$ & $\begin{array}{l}\text { Business } \\
\text { Domain }\end{array}$ & $\begin{array}{l}\text { Evaluation } \\
\text { activity }\end{array}$ \\
\hline $\begin{array}{l}\text { OntoDI } \\
\text { Methodology } \\
{[14]}\end{array}$ & $\begin{array}{l}\text { 6 steps } \\
\text { detailed } \\
\text { +complex( }\end{array}$ & $\begin{array}{l}\text { Good level } \\
\text { of Precision }\end{array}$ & $\begin{array}{l}\text { Data } \\
\text { integratio } \\
\mathrm{n}\end{array}$ & $\begin{array}{l}\text { Evaluation } \\
\text { +validation }\end{array}$ \\
\hline $\begin{array}{l}\text { Methontolgy } \\
{[15]}\end{array}$ & $\begin{array}{l}\text { Detailed } \\
\text { description }\end{array}$ & $\begin{array}{l}\text { Good level } \\
\text { of Precision }\end{array}$ & $\begin{array}{l}\text { Several } \\
\text { domains } \\
\text { (FIPA) }\end{array}$ & $\begin{array}{l}\text { Activity } \\
\text { (project } \\
\text { management } \\
\text { support } \\
\text { formalization }\end{array}$ \\
\hline
\end{tabular}

This comparative study results in the choice of the "Methontology" as a methodology for ontology elaboration. Indeed, "Methontology" is the most precised of all the previous methodologies. In addition, this methodology offers several types of activities and among these activities we mention project management.

\section{Ontology Validation Approaches}

The ontology validation is a very essential phase in the ontology construction process. Without this step, the ontology could not be exploitable or applicable. This stage becomes more and more complex with the increasing size of ontologies and the use of semantic construction [17].

Several approaches have been proposed in the literature for the validation of over ontology from different applications. In this section we will discuss the main approach of that have been proposed for the ontology validation.

\section{1) Main approaches of ontolygy validation}

a) An approach for validating the content of an ontology by a system based questions/answers [18]: Authors have proposed a semi-automatic approach called SAVANT based on the generation of questions to validate their ontologies. The first step is to automatically generate a list of Boolean questions from the ontology being validated. These questions are submitted to experts in the field who provide an agreement decision (Yes / No) and then an interpretation of these comments made to validate or modify the ontology. The originality of this approach rests on the fact that the interventions are manual and they are carried out only by health professionals.

b) An interactive method for the validation of ontology "OVIM" [19]: An ontology validation method called OVIM "Ontologies Validation by Interactive Method" has been proposed. Authors proposed this method for the structural and semantic validation of ontologies. This method will be based on five stages. They started with the structural validation that has four stages of validation namely; consistency, validation by OOps, validation by request and validation of the choice of the preferential label. In the fifth step they realized the semantic validation by collaborating with actors of the modeled domain.

c) An ontology validation Approach by the experts via a questionnaire [20]: An ontology evaluation and validation approach has been proposed. This approach starts from an ontology to be evaluated and ends with an ontology updated according to the evaluators' recommendations. The proposed approach consists essentially of five steps: In the first step a questionnaire is produced from the components of the ontology. Secondly, results of the survey of the experts will be done. The third step is to analyze and synthesize the results obtained. The update of the questionnaire based on expert feedback as well as the update of the ontology according to the knowledge of the results is realized during the last two stages.

d) A validation approach based on evaluation [21]: This approach essentially consists of verifying the consistency and measuring the impact of the change on the quality of the ontology. It also allows consistency checking and evaluation of the structure and content of the proposed ontology based on well-defined evaluation criteria and metrics.

\section{2) Discussion}

Although the validation approach proposed by [18] is a very important approach that allows the validation of concepts, relationships and axiom components of ontology, in fact it has been evaluated experimentally on three ontologies of different methods of construction but this approach presents some lacuna:

- A bad quality of validated ontology is related to two reasons:

1) The absence of a direct interaction between the ontology and experts to validate it [no interface].

2) Wrong time planning of the expert and the reduction of his level of concentration during the answers to the questions.

- The choice of questions is not generic; it also depends on the context of the problem.

- The validation method of [15] like any other method allows the structural and semantic validation. The problem here is that during the semantic validation domain actors verify only the existence of the general semantic domain.

- Another limit of this approach is the fact that the domain experts are not allowed to add, modify or update the used concepts.

- Expert, in this approach are simply domain actor and are not necessarily specialists in the field of ontology engineering.

- The approach proposed in [16], is a very interesting approach but has some limitations:

- It is an approach not updated in the term of the novelties of the version of the OWL language.

- Uses only English for the generation of questionnaires in natural language. 
- The questionnaires are generated using non-specialists in the construction of ontology study which reduces the quality of validated ontology.

The study of these different approaches allows us to notice that:

- A total absence of documentation.

- Absence of multi-expert validation [just one expert involved].

- Generally the major approaches make use simply of an evaluation of their ontologies. Effectively this evaluation could not be considered as a validation permitting to exploit really their ontology. It is in this context that we have proposed a so-called incremental validation approach which is mainly characterized by multi-intervention, documentation and incrementation. In the next section, we will describe both the process of building ontology and the proposed validation.

\section{ONTO-COMPUTER-PROJECT: METHODOLOGY OF CONSTRUCTION}

The proposal of a knowledge capitalization approach is the main goal of our current research study. This approach consists essentially of two processes: a knowledge acquisition and formalization process and a support decision for project management process.

The present paper is only concerned by the first process. It is composed of two phases: The phase of acquisition and the phase of formalization of knowledge. This process is relayed by the proposal of domain ontology wish structures and organizes the great mass of the concepts and knowledge encapsulated in the proposed models.

In a previous work [5] we proposed an ontological construction approach based on the methodolgy "Methontolgy" which leads to a first version of our domain ontology. In this paper, we will:

First detail the description of this approach, by applying carefuly the methodology "Methontolgy" wich has been select in the bases of a comparative study i..e 2.1.2. Finaly, we present a recent version of the ontology [Fig. 6] with our proposed validation approach. The particularity of "Methontolgy" is the possibility of the return on the steps preceding. In what follows, inspired from "Metontology", we will present the stages of the construction of our domain ontology:

- Step1: this step consists in building a glossary of terms containing all the domain knowledge that is useful and potentially usable for the construction of computer domain ontology. This glossary includes concepts, instances, verbs and attributes. To do this step, we have met with domain specialists and experts to talk about computer projects.

- Step2: "classes and class hierarchy": In this step we have built taxonomies of concepts and terms obtained via the grouping, the categorization and the generalization of the different concepts studied [Fig. 1].
- Step 3: "relations between classes" During this stage we have created the relations between classes by determining for each relation the type of relation and the classes to be connected [Fig. 2].

- Step 4: the instantiation of this ontology [individuals + instances] is actually the new case on which our reasoning is based [Fig. 4].

- Step 5: This step provides a detailed description of previously identified relationships, attribute concepts, and constants. We have used the projects of the company and the structure of documents to define some classes and some attributes.

- Step6: "Data properties" It concerns the description of formal rules and axioms relating to the various elements of ontology already known. [Fig. 3].

- Step 7: This steps concerning the detailed description of instances and relations between instances, classes and properties [Fig. 5].

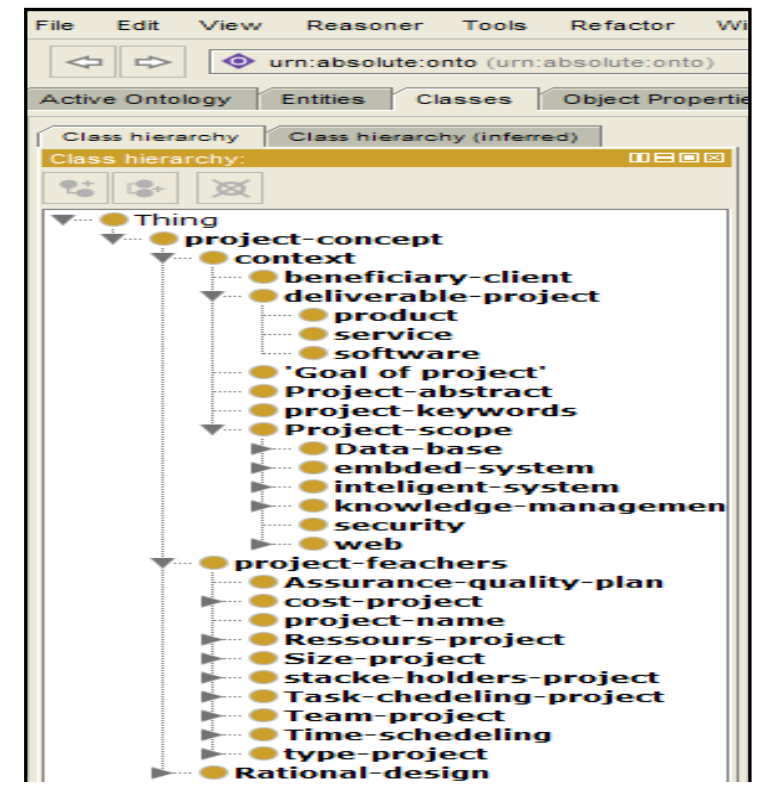

Fig. 1. Class Hierarchy.

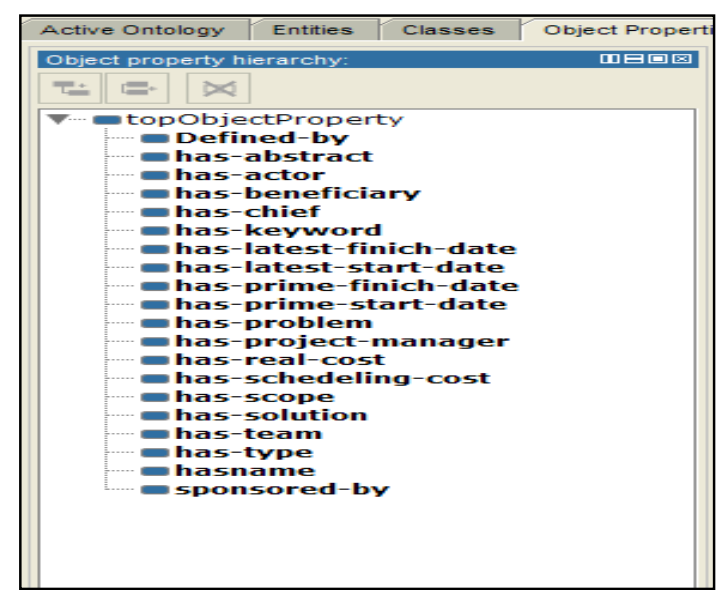

Fig. 2. Object Properties. 


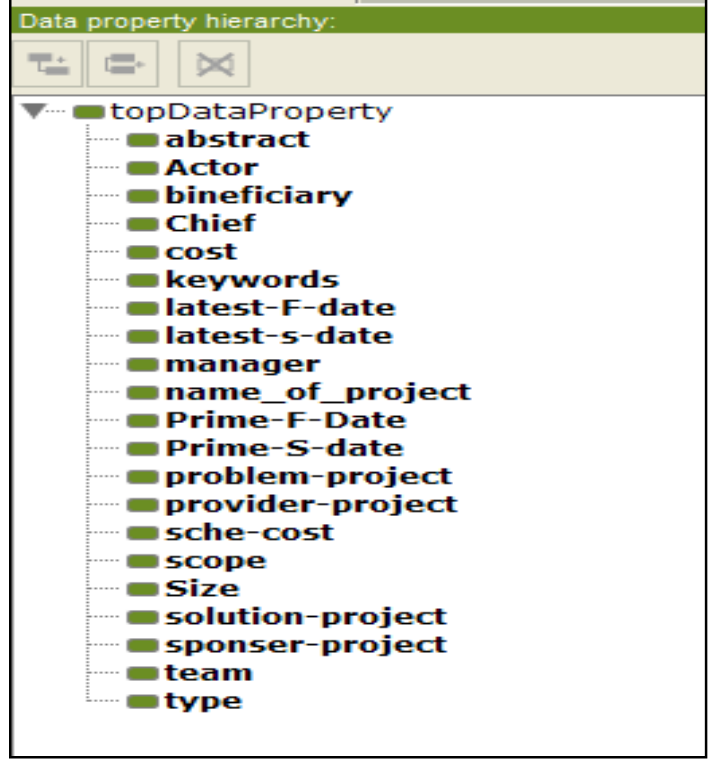

Fig. 3. Data Property of Ontology.

\begin{tabular}{|l|l|}
\hline Individuals: \\
\hline actor1 \\
beneficiaryc1 \\
chief 1 \\
keywords1 \\
latest-start-date1 \\
latst-finich-date1 \\
manager1 \\
prime-finich-date1 \\
prime-start-date1 \\
problem 1 \\
project1 \\
projectabstract1 \\
projectname1 \\
projectscope1 \\
provider1 \\
real-cost1 \\
shedeling-cost1 \\
size1
\end{tabular}

Fig. 4. Example of Instantiation.

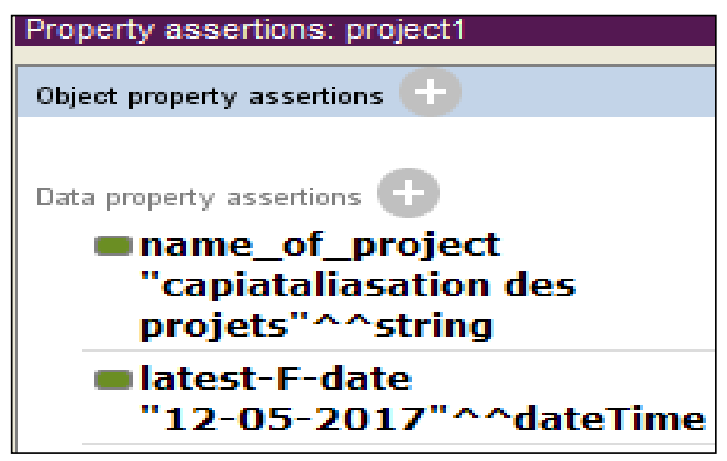

Fig. 5. Relation between Instances.

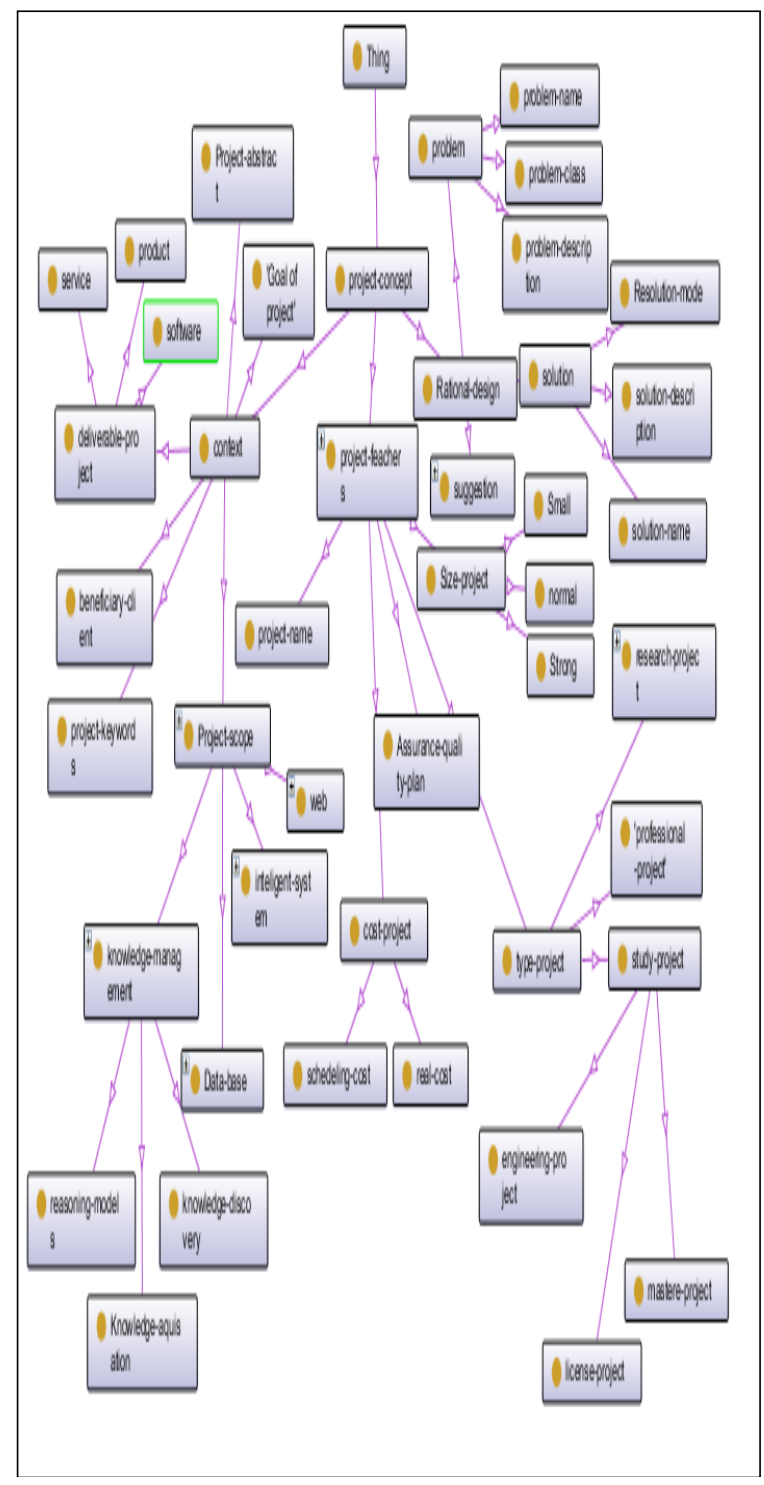

Fig. 6. Onto-Computer-Project: Final Computer Domain Ontology Version.

\section{INCREMENTAL AND MULTI-INTERVENTION VALIDATION APPROACH}

Evaluating ontology means checking and validating two aspects: structural aspect and semantic aspects. The validation of the structural aspect of ontology allows verifying the consistency and the coherence of a model to check. In this way, classes and sub-classes are verified according to criteria of consistency and coherence between them and to avoid redundancy.

The validation of the semantic aspects involves communication aspects between actors of different domains of expertise. In this way, we proposed a validation approach based on three criteria:

- The first criterion: the Incremental validation of the ontology: the passage from one validation step to another results in an update [modification, deletion or addition] of the initial ontology. 
- The second criterion: the Multi-intervention criteria: This approach is characterized by the intervention of several and different experts. Three experts are involved in the validation process:

$\checkmark$ The project management expert: He is an expert in the field of project management.

$\checkmark$ The project computer expert: $\mathrm{He}$ is an expert who masters all the concepts of computer projects.

$\checkmark$ The specialist in ontology engineering: this actor has a good command of all the tools and editors of the ontology.

- The third criterion: our validation approach is respecting the "V cycle". We inspired by the live cycle of software engineering. Effectively our approach like the $\mathrm{V}$ cycle requires a feedback between all the validation phases. Hence, in our validation phases we can return to any expert for revalidation if needed. In contrary of a classic approach which applied semantic and structural validation definitively with no return, we can return at any phase validation to enhance our ontology. The approach that we proposed is essentially composed of six steps [Fig. 7]:

- Step 1: During the first validation step, a descriptive document presented in tabular form containing all the concepts and terms as well as their descriptions constituting our first version of the ontology was be prepared.

- Step 2: In the second step, it is up to us to update our proposal based on the remarks and the assertions given by the computer project expert. This step was considered as a meeting accompanied by discussions. The result of this phase is a second ontology's version that is ready to be evaluated by "project computer expert". This version is an amelioration of the version 1 at the level of project features [Fig. 8].

- Step 3. During this step, we prepare a second report: a document describing our objectives and orientations. This report is then submitted to a project management expert for evaluation. This second expert could affirm or refute, add or modify the proposal by adding a textual justification. Effectively, in a version 3, this expert proposes to restrict the ontology by adding a new super class named "project context". This class gives a detailed idea about "project deliverables", "project abstract" and "project keywords", etc. [Fig. 9].

- Step 4: After the evaluation done by the project management expert, we have to do at this present step a technical check this check makes use of a software tool in the way to evaluate the consistency and the coherence of the latest version of our ontology. This mission is assured by a specialist in ontology engineering and results in a version 4.

- Step 5: at this step, the version 4 is sent to the project management expert according to our objective which is essentially to discuss about projects problem solving. Our goal here is to enrich ontology in the way to facilitate problems solve in a new project by exploiting historical projects. This step leads to a new version of ontology labeled version 5. At this effect the expert proposes to add a new sub-class baptized "Rational design" [Fig. 10].

- Step 6: For this validation phase the specialist of ontology engineering chooses to use HERMIT [tool integrated in protégé 4.2] to validate the consistency. This step results in a new version 6 .

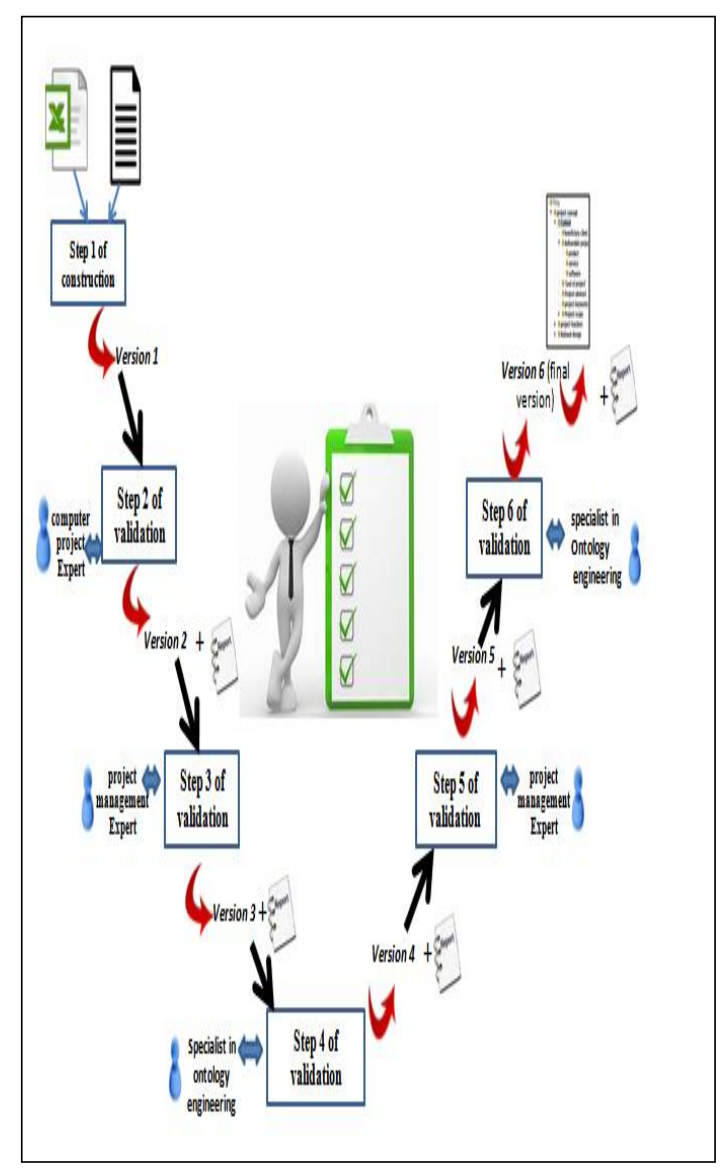

Fig. 7. Incremental Validation Approach.

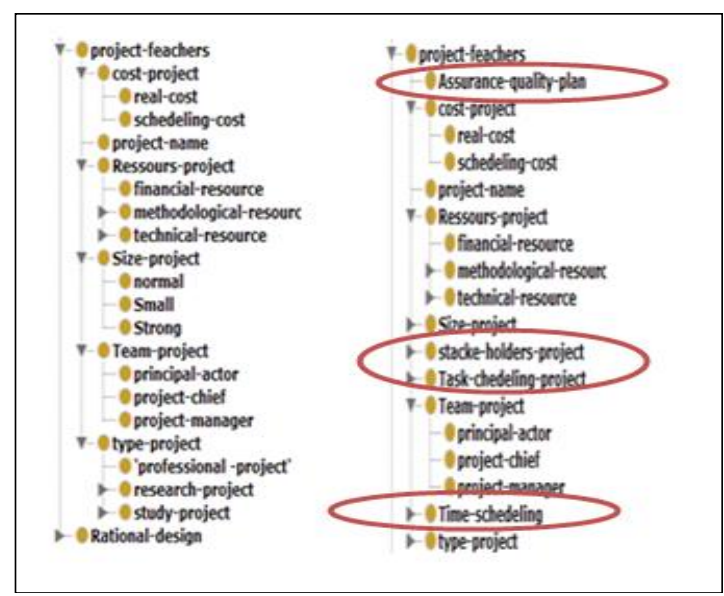

Fig. 8. Updating of Project Features. 


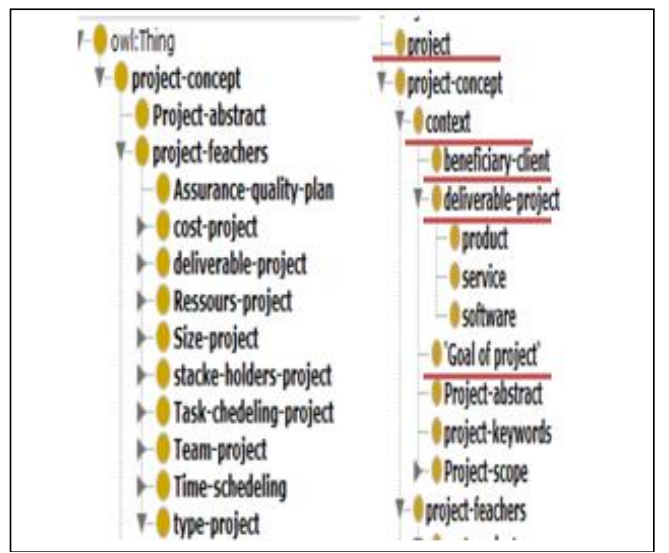

Fig. 9. Adding of Project Context Class.

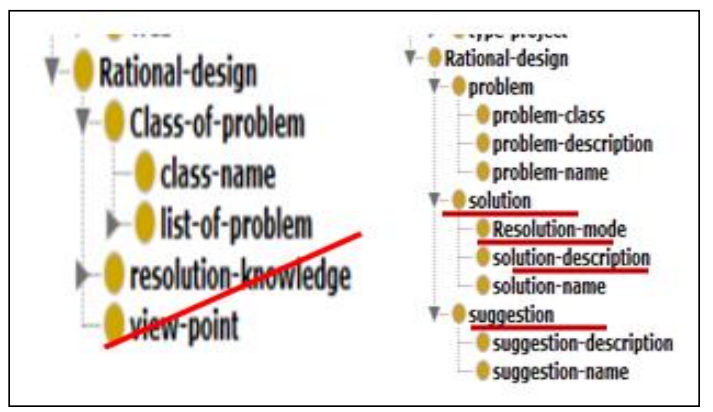

Fig. 10. Rational Design Class Update.

\section{CONCLUSION AND PERSPECTIVES}

Validation ontology plays an important role during the creation and updating of ontology to obtain a final and suitable ontology version. In this paper we have proposed two approaches: an ontology construction approach and a validation approach.

The construction of this domain ontology allowed us to have a complete idea on the concept of projects and specifically computer projects. It also provides support or help for users to acquire new projects that need to be classified. The proposed validation approach is an incremental and a multi-intervention approach that allows a semantic and structural validation of the proposed ontology.

After the validation phase we will validate experimentally this ontology. It is in this context that our near future work will be focused on the experimentation phase. This phase is carried out by building a knowledge base containing a real computer projects forming the basis of the facts and a set of rules forming the basis of the rules. These rules are of two types: classification rules which help to classify the projects and association rules which provide a help to describe in detail a new project.

To do this, we will use the classification data mining techniques and we are going to propose classification and learning algorithms.

\section{REFERENCES}

[1] B.Menaouera, S.Khalissab , B.Abdelbakic , T.Abdelhamidd "Towards a new approach of support innovation guided by knowledge management:
Application on FERTIA, 4th International Conference on Leadership, Technology, Innovation and Business Management, 2015.

[2] M.Hemam1 et al, An Ontological Approach for Domain Knowledge Modeling and Querying in Wireless Sensor Networks, The 2nd International Conference on Pattern Analysis and Intelligent Systems, PAIS, 2016.

[3] D.Monticolo et al, An agent-based system to build project memories during engineering projects, Knowledge-Based Systems, 2014.

[4] F. Belkadi, E. Bonjour, M. Camargo, N. Troussier, B. Eynard, A situation model to support awareness in collaborative design, Int. J. Hum.-Comp. Stud. 71 (1), 2013.

[5] H.Raja, M.Lassad, BH.Henda,"Computer-Project-Ontology Construction,Validation and choice of knowledge base",10th International Joint Conference on Knowledge Discovery, Knowledge Engineering and Knowledge Management, 2018.

[6] K. Drame, "Contribution to ontology construction and information retrieval: application to the medical field," thesis, Université de Bordeaux, 2014.

[7] M.Harzallah,Contributions to Knowledge Engineering: Construction and Validation of Ontology and Semantic Measurements, Habilitation to Direct Research (H.D.R.),2017.

[8] A.Amarir, El.Benlahmer, L.el houssine, "The methods of building ontology from text", Conference Paper, The second day on Information Technologies and Modeling TIM'14, May 2014.

[9] G.Enrico.Caldarola, M.Antonio.Rinaldi, "An Approach to Ontology Integration for Ontology Reuse IEEE 17th International Conference on Information Reuse and Integration, At Pittsburgh, Pennsylvania, 467 Reads. with USA, July 2016.

[10] J.Gherasim, M.Harzallah , G. Berio, P. Kuntz, "Comparative analysis of methodologies and tools automatic ontology construction from textual resources, *LABSTICC, UMR 3192 CNRS,2013.

[11] E. Mezghani, E. Exposito, K.Drira, A collaborative Methodology for tacit knowledge management: Application to scientific research, Future Generation Computer Systems, 2015.

[12] Y.Sure,S.Staab,Rudi Studer,On-To-Knowledge Methodology [OTKM], Institute AIFB, University of Karlsruhe Postfach, 76128,Karlsruhe, Germany,http://www.aifb.unikarlsruhe.de,2004.

[13] M.Groninger,M.S.Fox, The Role of Competency Questions in Enterprise Engineering, Department of Industrial Engineering, University of Toronto, 4 Taddle Creek Road, Toronto, Ontario M5S IA4, 2017.

[14] A.Yunianta et al, Methodology for Ontology Development on Data Integration (OntoDI), (IJACSA) International Journal of Advanced Computer Science and Applications, 2019.

[15] H. Qiua,d , G.F. Schneidera,d , T. Kauppinenb , S. Rudolphc S. Steigerd, Reasoning on Human Experiences of Indoor Environments using Semantic Web Technologies,35th International Symposium on Automation and Robotics in Construction, 2018.

[16] L. Trouche, S. Aubin, V. Soulignac, L. Guichard. Construction of a semantic model to organize knowledge dedicated to agroecology. The case of Agro-PEPS / GECO. Agronomy, Environment and Societies, French Association of Agronomy, 2016.

[17] M.Harzallah, Contributions to Knowledge Engineering:Construction and Validation of Ontology and Semantic Measures, Habilitation to Direct Research (H.D.R.),2017.

[18] Ben Abacha et al.,Towards Natural Language Question Generation for the Validation of Ontologies and Mappings, Journal of Biomedical Semantics, 2016.

[19] M.Richard, X.Aimé, M.Krebs, J.Charlet, LOVMI: towards an interactive method for the validation of ontologies, INSERM UMRS 1142, LIMICS, F-75006, 2015.

[20] G.Leila,M.aya,D.faiza, generation of a questionnaire from a domain ontology,conference paper, 2017.

[21] S.tartir,S.Amit,IA.Young,Ontological Evaluation and Validation, from book Theory and Applications of Ontology: Computer Applications [pp.115-130], 2010. 